\title{
High Brightness Photocathodes for Ultrafast TEM: A New Paradigm
}

\author{
B.L. Rickman, ${ }^{1}$ J.A. Berger, ${ }^{1}$ A.W. Nicholls, ${ }^{2}$ and W.A. Schroeder ${ }^{1}$
}

${ }^{1}$ University of Illinois at Chicago, Department of Physics (m/c 273), 845 W. Taylor Street (rm. 2236), Chicago, IL 60607-7059

${ }^{2}$ Research Resources Center, University of Illinois at Chicago (m/c 337), 845 W. Taylor Street, Chicago, IL 60607

Dynamic transmission electron microscopy (DTEM) aims to combine the high spatial resolution of electron microscopy with the temporal resolution afforded by pulsed laser systems [1]. Key to the development of a viable ultrafast (i.e., sub-nanosecond) TEM is a laser-driven photoemission source with a high brightness; that is, a low normalized transverse emittance, $\varepsilon_{\mathrm{T}}=\Delta x . \Delta p_{T} /(m c)$, where $\Delta x$ and $\Delta p_{T}$ are the spatial size and transverse rms momentum of the source respectively. Whereas $\Delta x$ is limited by Gaussian laser beam focusing and Child's Law, $\Delta p_{T}$ is dependent upon the properties of the photoelectron emitter. For photocathodes at a thermal energy $k_{\mathrm{B}} T$ much less than the excess photoemission energy $\hbar \omega-\phi_{\text {eff, }}$, where $\hbar \omega$ is the incident photon energy and $\phi_{\text {eff }}$ is the effective work function, the standard expression for $\Delta p_{T}$ is $\sqrt{m\left(\hbar \omega-\phi_{\text {eff }}\right) / 3}$ [2], where $m$ is the electron mass. Extensive simulations of the photoemission process and detailed experimental investigations of several planar metal photocathodes indicate that this expression is incomplete: the mean square transverse momentum should be written as

$$
\left(\Delta p_{T}\right)^{2} \approx \frac{M\left(\hbar \omega-\phi_{\text {eff }}\right)}{3} \sqrt{1+\left(\frac{3 k_{B} T_{e}}{\hbar \omega-\phi_{\text {eff }}}\right)^{2}},
$$

where $T_{e}$ is the photocathode electron temperature and $M=\min \left(m_{T^{*}}, m_{0}\right)$, with $m_{T}{ }^{*}$ being the transverse electron effective mass of the state (i.e., energy band) from which the electron is emitted and $m_{0}$ is the electron rest mass. For $m_{T}^{*}$ less than $m_{0}$, the electron beam brightness is therefore proportional to $\left(m_{T}^{*}\right)^{-1}$ - defining a new avenue for the future development of high brightness laserdriven pulsed electron sources.

The results of our photoemission simulations illustrated the cause for the dependence of $\Delta p_{T}$ on $m_{T}{ }^{*}$. Shown in Figure 1 are two photoemission efficiency contour plots (as a function of the longitudinal $\left(p_{z}\right)$ and transverse $\left(p_{T}\right)$ emission momenta) for Mo $\left(\phi_{\text {eff. }}=4.50( \pm 0.05) \mathrm{eV}\right)$ when $\hbar \omega=4.75 \mathrm{eV}$ and $T_{e}$ $=300 \mathrm{~K}$; Fig.1(a) for $m_{T}{ }^{*}=m_{0}$, and Fig.1(b) for $m_{T}{ }^{*}=0.3 m_{0}-$ a value of the electron effective mass extracted from cyclotron resonance studies of Mo [3]. Clearly evident is that energy and momentum conservation limit the maximum electron emission angle for $m_{T^{*}}<m_{0}$; specifically, $\theta_{\max }=\sin ^{-1} \sqrt{m_{T}^{*} / m_{0}}$ [4], which is $33^{\circ}$ for $m_{T}^{*}=0.3 m_{0}$. This then causes a reduction in the extracted value of $\Delta p_{T}$ from $0.29 \sqrt{ }\left(m_{0} . \mathrm{eV}\right)$ for $m_{T}^{*}=m_{0}$ to $0.16 \sqrt{ }\left(m_{0} . \mathrm{eV}\right)$ when $m_{T}^{*}=0.3 m_{0}$.

Figure 1(c) displays the experimental results obtained for a $300 \mathrm{~K}$ planar Mo photocathode in our $20 \mathrm{kV}$ photo-electron gun driven by $4 \mathrm{ps}$ duration $261 \mathrm{~nm}(\hbar \omega=4.75 \mathrm{eV}) \mathrm{UV}$ laser pulses [5]. After acceleration, the electron pulses directed down the 'optical axis' of a pair of large-aperture, round magnetic lenses before detection using a YAG scintillation screen and a CCD camera. Their spatial 
spot size on the YAG scintillator is monitored as a function of the magnetic lens strength (i.e., the square of the current in the lens coils) and compared to a simulation of the measurement technique that employs an extended analytical Gaussian (AG) electron pulse propagation model [6]. The experimental data points are in good agreement with the simulated propagation of an electron pulse with $\Delta p_{T} \approx 0.15 \sqrt{ }\left(m_{0} . \mathrm{eV}\right)$ rather than for $\Delta p_{T}=0.29( \pm 0.03) \sqrt{ }\left(m_{0} . \mathrm{eV}\right)$ (the shaded region in Fig.1(c)) which would be expected for $m_{T}^{*}=m_{0}$; thus, providing clear evidence supporting the proposed dependence of $\Delta p_{T}$ on $m_{T}^{*}$.

\section{References:}

[1] W.E. King, et al., J. Appl. Phys. 97 (2005) 111101.

[2] D.H. Dowell and J.F. Schmerge, Phys. Rev. ST - Acc. \& Beams 12 (2009) 074201.

[3] M. Surma, J. Mag. \& Mag. Mat. 11 (1979) 56.

[4] Zhi Liu, et al., J. Vac. Sci. Tech. B 23 (2005) 2758.

[5] J.A. Berger, et al., Appl. Phys. Lett. 101 (2012) 194103.

[6] J.A. Berger and W.A. Schroeder, J. Appl. Phys. 108 (2010) 124905.

[7] This work was supported by the Department of Energy (contract DE-FG52-09NA29451).

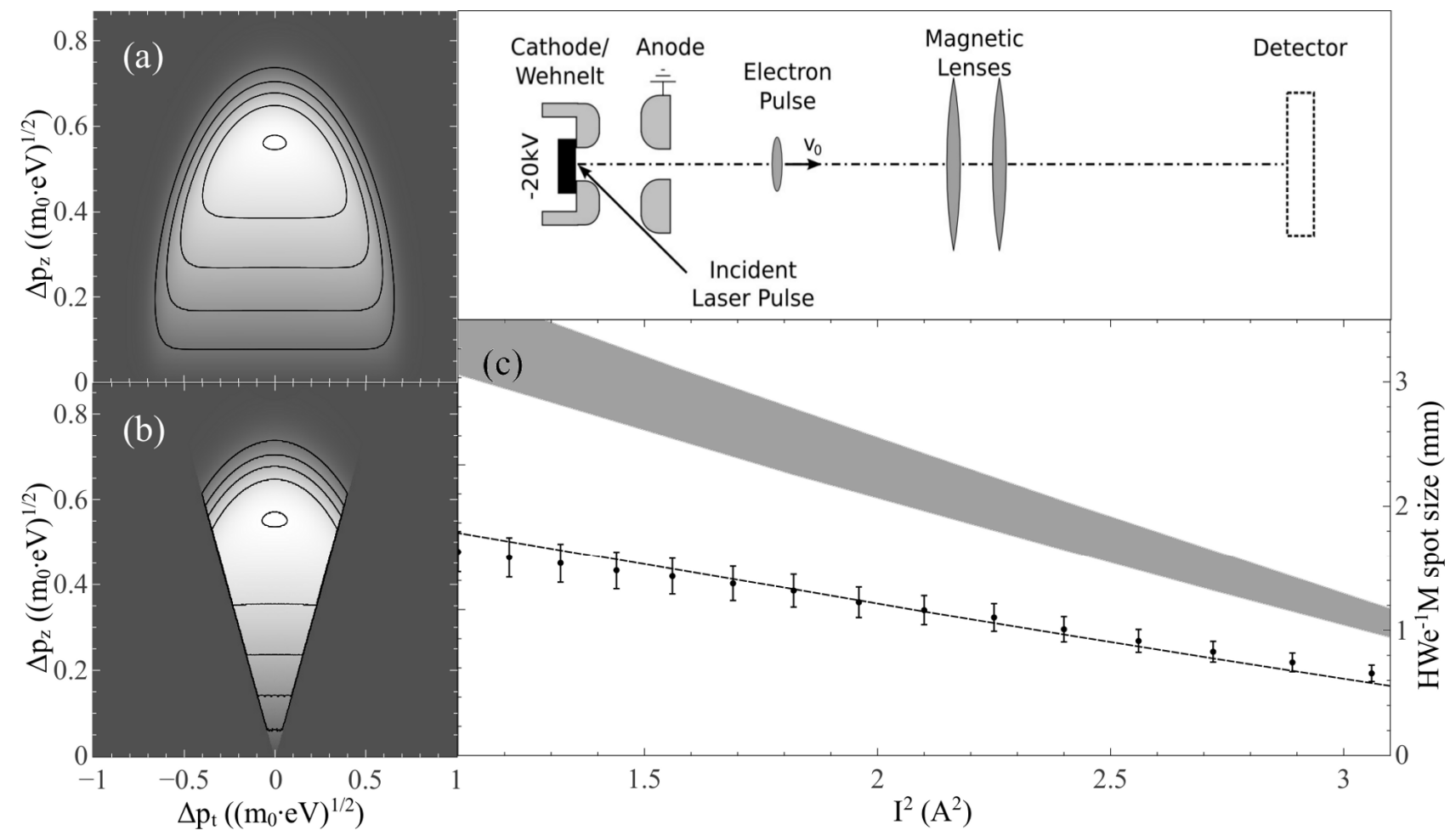

Figure 1. Photoemission from metal photocathodes. Theoretical emission momentum contour plots (longitudinal $\left(p_{z}\right)$ versus transverse $\left.\left(p_{T}\right)\right)$ for a Mo photocathode $\left(\phi_{\text {eff. }}=4.50 \mathrm{eV}, \hbar \omega=4.75 \mathrm{eV}\right.$, and $T_{e}$ $=300 \mathrm{~K}$ ) for (a) $m_{T}^{*}=m_{0}$ and (b) $m_{T} *=0.3 m_{0}$. (c) Observed electron beam spot size as a function of magnetic lens strength (square of coil current) for a Mo photocathode; AG model simulations for $\Delta p_{T} \approx 0.15 \sqrt{ }\left(m_{0} . \mathrm{eV}\right)$ (dashed line) and $\Delta p_{T}=0.29( \pm 0.03) \sqrt{ }\left(m_{0} . \mathrm{eV}\right)$ (the shaded region). A schematic of the experiment is shown top right. 